\title{
Case Report \\ A Case of Diffused Malignant Pleural Mesothelioma Forming Small Multiple Disseminations with Intraoperatively Suspicious Carcinoid Tumors
}

\author{
Ryuichi Suemitsu, MD, PhD, ${ }^{1}$ Sadanori Takeo, MD, PhD,${ }^{1}$ Motoharu Hamatake, MD, $\mathrm{PhD},{ }^{1}$
}

Kiyomi Furuya, $\mathrm{MD}, \mathrm{PhD}{ }^{2}$ and Noriko Uesugi, $\mathrm{MD}, \mathrm{PhD}^{3}$

\begin{abstract}
A 65-year-old male, having symptoms suggestive of pulmonary malignant tumor, underwent video-assisted thoracic surgery (VATS). Surgery revealed a solid tumor originating from the thoracic wall, with many small solid tumors in the thoracic wall and diaphragm near the tumor. The intraoperative observation of a frozen section typed the tumor as carcinoid; however, hematoxylin-eosin staining and immunohistological findings provided the definitive diagnosis of diffused, malignant pleural mesothelioma (MPM).
\end{abstract}

Key words: malignant pleural mesothelioma; epithelioid type; dissemination; video-assisted thoracoscopic surgery

\section{Introduction}

Malignant pleural mesothelioma (MPM) is an uncommon neoplasm that is usually associated with asbestos exposure. Pathologically, these tumors are classified as diffused MPM, localized MPM and others. ${ }^{1)}$ localized types are a single lesion, and diffused types, multiple lesions. However, it is difficult to differentiate the morphologies of early-phase tumors. In the past, localized mesothelioma was defined as a solitary fibrous tumor of mesenchymal stem cell origin, distinguished from localized mesothelioma. There are few reports of early-stage

${ }^{1}$ Department of Thoracic Surgery, Clinical Research Institute, National Hospital Organization, Kyushu Medical Center, Fukuoka, Fukuoka, Japan

${ }^{2}$ Department of Radiology, Clinical Research Institute, National Hospital Organization, Kyushu Medical Center, Fukuoka, Fukuoka, Japan

${ }^{3}$ Department of Pathology, Clinical Research Institute, National Hospital Organization, Kyushu Medical Center, Fukuoka, Fukuoka, Japan

Received: January 23, 2009; Accepted: March 15, 2010

Corresponding author: Ryuichi Suemitsu, MD, PhD. Department of Thoracic Surgery, National Hospital Organization, Kyushu Medical Center, Jigyohama 1-chome, 8-1, Chuo-ku, Fukuoka, Fukuoka 810-8563, Japan

Email: ryuichi_shizuka@yahoo.co.jp

(C2011 The Editorial Committee of Annals of Thoracic and Cardiovascular Surgery. All rights reserved.
MPM or details about its forming a small, multiple nodular pattern or dissemination of the tumor.

\section{Case Report}

A 65-year-old male presented with a continuous cough. Chest computed tomography (CT) was taken by his family physician. Chest CT showed homogeneous multiple tumors in the S10 region of the left lower lobe, $3.5 \mathrm{~cm}$ in diameter, lining the thoracic wall (Fig. 1A and 1B) and there were no lesions in any of the other lobes, including the mediastinum. Positron emission tomography (PET) revealed a small hot spot in these pulmonary tumors, and its maximum standard uptake value was 2 $\mathrm{mg} / \mathrm{mL}$. Fiber optic bronchoscopy revealed a normal bronchus, and a biopsy of the tumor revealed no malignancy. Since there was a chance that the lesion might be malignant, we preformed extirpation by video-assisted thoracic surgery (VATS). Thoracoscopy showed a tumor on the diaphragm and the cardio-phrenic angle. Furthermore, multiple small nodular lesions of no more than 10 tumors were observed not only on the diaphragm but also on the lower thoracic wall (Fig. 1C and 1D). The intraoperative observation of a frozen section contained many monotonous cells with medium sized nuclei and small nucleoli arranged in sheets, trabeculae and small acini. There was no evidence of Mitotic figures or 


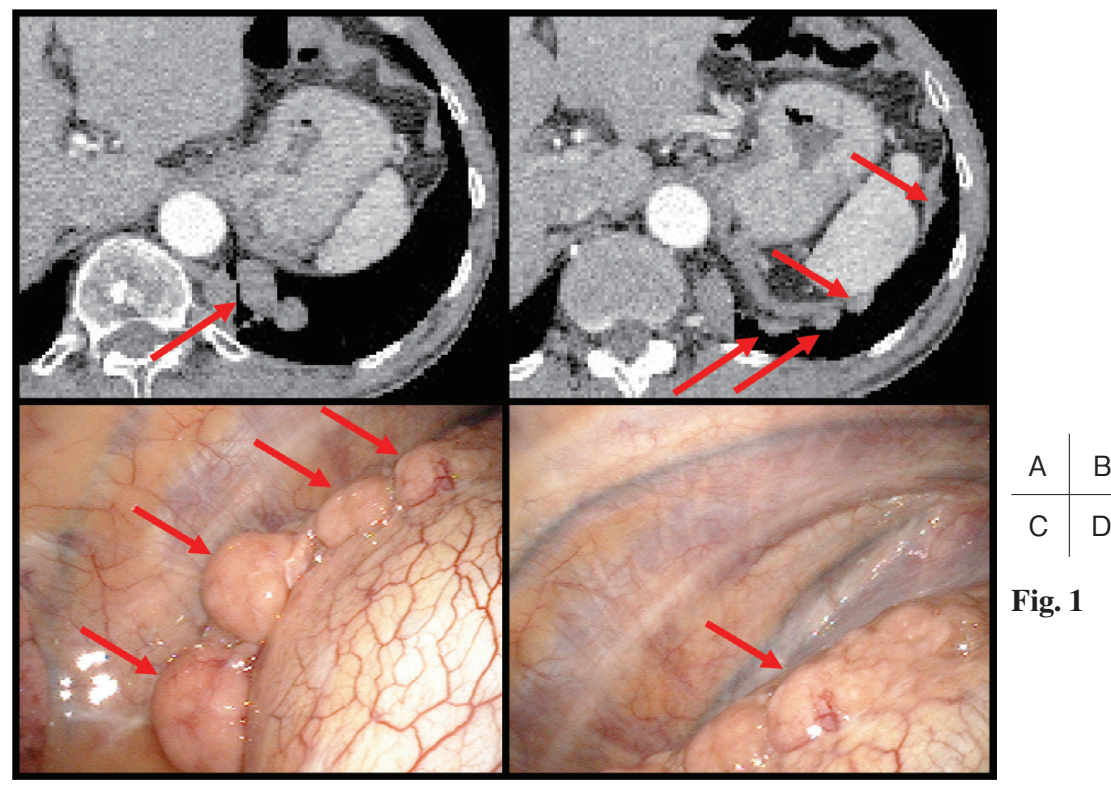

(A and B) Chest CT of a homogeneous solid tumor and multiple small nodules on the thoracic wall. (C and D) Surgical findings. Multiple solid tumors on the thoracic wall and diaphragm. The visceral pleura had no abnormal findings.

necrosis. We suspected the tumors to be carcinoid; thus, we resected the entire area, including the extrapleura and part of the diaphragm. Diffused MPM was the postoperative diagnosis, from the hematoxylin-eosin staining (Fig. $\mathbf{2 A}$ and 2B) and immunological findings (Fig. 2C-2F). The morphology of these tumors was epithelioid, without sarcomatoid components. Furthermore, mesothelial markers, such as Calretinin and D2-40 were all positive, and TTF-1, a negative mesothelial marker, was not detected. We had expected a single lesion without diffuse invasion for localized MPM, thought the diagnosis here was diffused MPM of type epithelioid. The patient has returned to his usual activities and has remained well for 19 months since the operation without any evidence of recurrence or metastasis.

\section{Discussion}

Diffused MPM is a distinct malignant tumor of the pleura that arises from pluripotential mesothelial cells. In 1931, Klemperer et al. classified mesothelioma into epithelial-carcinomatous, mesenchymal-sarcomatous and mixed biphasic subgroups. ${ }^{2}$ In the WHO classification, mesotheliomas are divided into 3 groups: diffused malignant mesothelioma, localized malignant mesothelioma and other tumors of mesothelial origin.") The staging of this case would be Ia (pathological T1a, clinical N0, clinical M0) according to the International Mesothelioma Interest Group (IMIG); however, the TNM classification does not clearly define diffused MPM when there is dis- semination, localized on the thoracic wall and diaphragm. ${ }^{1,3)}$ Localized MPM is relatively rare, and it is characterized by uncommon, sharply circumscribed tumors of seromal membranes with the microscopic appearance of diffuse malignant mesothelioma, but without any evidence of diffuse spread. ${ }^{1,4)}$ In this case, multiple nodular tumors localized on the thoracic wall and diaphragm were diagnosed as diffused MPMs and not specified as localized MPM because of the multiple lesions. Localized MPMs are characterized by a single presentation; furthermore, there are no reports with regard to multiple localized MPM. There is also no definition of multiple localized MPM. If the lesion in this case had been a single tumor, it would be diagnosed a localized MPM. This case was assumed to be an early phase of diffused MPM with multiple small lesions originating as disseminations, lymphatic vessel metastases or vascular metastases. Dissemination is the best explanation for multiple small lesions in this case, because the excision surface of each resected tumor had no intravascular or intralymphatic invaded tumor cells. There is one report of an early localized peritoneal mesothelioma with dissemination $^{5}$; however, there are no reports of the dissemination of primary small size MPM. Though the multiple diffused MPMs were not confirmed to be dissemination, it is a certainly rare case and the accumulation of reports such of rare MPMs will allow clarification of the occurrence of this disease.

With respect to surgery in this case, preoperatively we could not diagnose a malignancy. We had undergone 


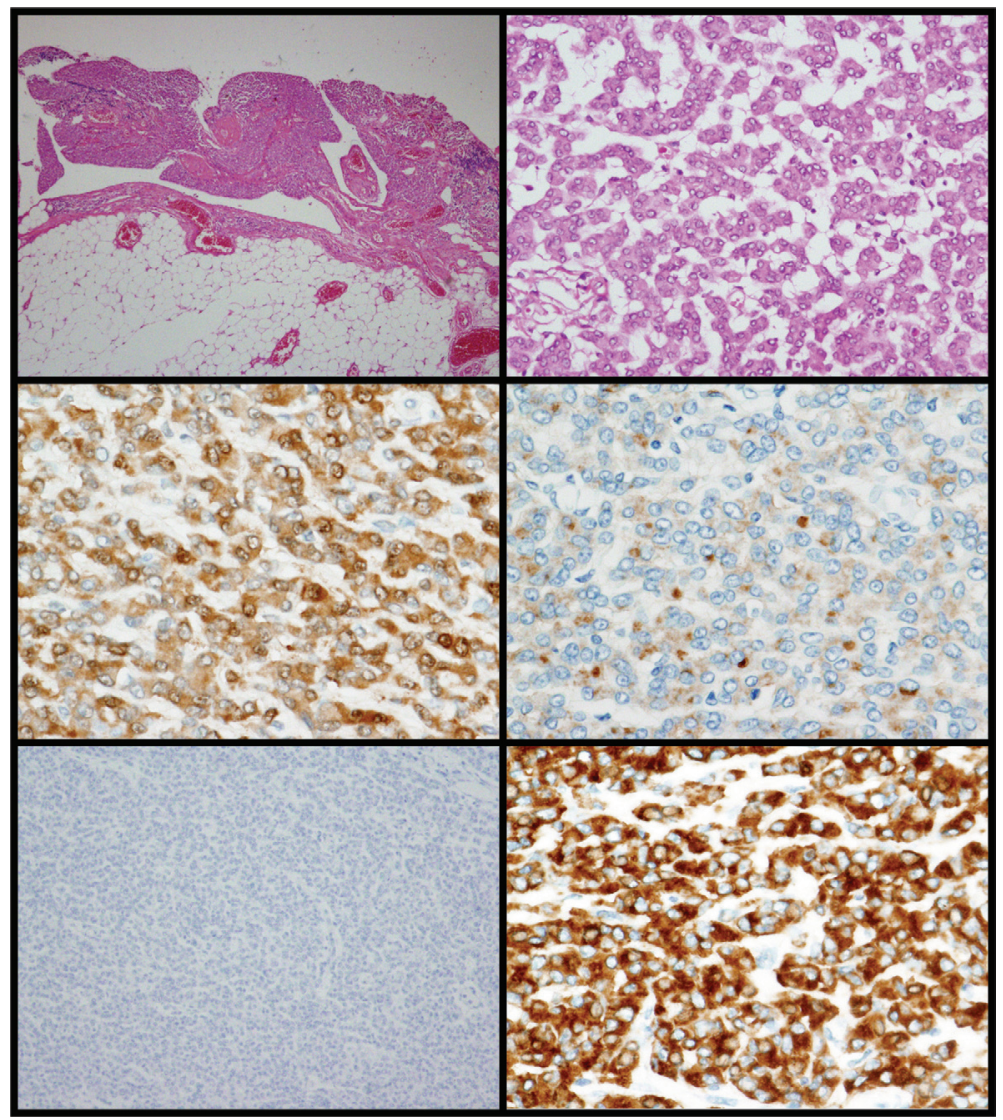

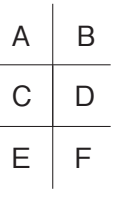

Fig. 2 (A and B) Microscopic finding with hematoxylin and eosin stain: (A) High power view and (B) Low power view. The cells have eosinophilic cytoplasm with open nuclei. Epithelioid forms are deceptively monotonous and bland in appearance. There are few mitotic cells. (C-F) Immunohistological findings of the tumor (light microscope): Immunohistological positive markers of malignant mesothelioma such as Calretinin (C) and D2-40 (D) were positive and negative marker TTF-1 (E) was negative; AE1/AE3 (F) is an epithelial marker and it was also positive. The tumor was consequently diagnosed as a malignant pleural mesothelioma of the epithelioid type. surgery including an extirpation. Intraoperatively diagnosed by a frozen section, we had diagnosed carcinoid tumors. We tried to resect all visible tumors because of two reasons. One reason is that carcinoid tumors have a potentiality of long-term survival if a complete resection is possible. Another is that carcinoid tumors usually have tolerances to chemotherapy and radioactivity, so we have judged surgical resection to be the best treatment for this patient. However, as a result of paraffin fixed sections after surgery, a final diagnosis was diffused MPM. Surgical indication of epithelioid type MPM with stage I or II would usually be considered to be pleurectomy or extrapleural pneumonectomy, ${ }^{6}$ and we might perform a second-look operation if the patient does not refuse. After 10 months, the patient had no heath problems, and his chest CT showed no recurrence. Extremely early phase MPM, such as this case, is most likely cured after resection by VATS; however, we continue to would follow-up this patient over the long term.

\section{Referencese}

1) Travis WD, Brambilla E, Muller-Hermelink HK, Harris CC. WHO Classification of Tumours. Pathology and Genetics of Tumours of the Lung, Pleura, Thymus and Heart. Lyon: International Agency for Research on Cancer (IARC) Press 2004; pp 125.

2) Klemperer P, Rabin CB. Primary neoplasms of the pleura. Arch Pathol Lab Med 1931; 11: 385-412.

3) Rusch VW. A proposed new international TNM staging system for malignant pleural mesothelioma. From the International Mesothelioma Interest Group. Chest 1995; 108: 1122-8.

4) Allen TC, Cagle PT, Churg AM, Colby TV, Gibbs AR, et al. Localized malignant mesothelioma. Am J Surg Pathol 2005; 29: 866-73.

5) Sugarbaker PH, Yan H, Grazi RV, Shmookler BM. Early localized peritoneal mesothelioma as an incidental finding at laparoscopy. Report of a case and implications regarding natural history of the disease. Cancer 2000; 89: 1279-84.

6) Sugarbaker DJ, Flores RM, Jaklitsch MT, Richards WG, Strauss GM, et al. Resection margins, extrapleural nodal status, and cell type determine postoperative long-term survival in trimodality therapy of malignant pleural mesothelioma: results in 183 patients. J Thorac Cardiovasc Surg 1999; 117: 54-65. 